\title{
Patella as an osteoarticular autograft for reconstructing the articular surface of knee after osteosarcoma in the proximal end of tibia: a case report
}

\author{
Amit Kumar Seth ${ }^{1}, *$ Tao Sheng Xiang ${ }^{1}$ \\ I'Department of Orthopedic Surgery, Zhong Nan Hospital/ Wuhan University, Hubei, China) \\ *(Corresponding Author)
}

\begin{abstract}
Osteosarcoma is the most common histologicalform of primary bone cancer. It is an aggressive malignant neoplasm arising from primitive transformed cells of mesenchymal origin that exhibit osteoblastic differentiation and produce malignant osteoid. The most common form of treatment is removal of the lesion. Limb-sparing procedures can often be used to preserve function. Chemotherapy is also required to treat micrometastatic disease, which may be present, but not detectable in most patients at initial diagnosis.

We present a proximal anteromedial tibial metaphysical Osteosarcoma in a 51 year-old adult male. Diagnosis was confirmed by radiography and histopathology. Pre-up palliative care, en bloc resection of the tumor with the patella and iliac graft reconstruction and post-operative chemotherapy were performed. After multi-step surgeries, the patient made full clinical and symptomatic recovery. Further 4 and 10 years follow ups revealed a promising outcome.
\end{abstract}

Keywords: Autograft, knee, Osteoarticular Autograft, Osteosarcoma, patellar grafting

\section{Introduction}

Osteosarcoma has a bimodal age distribution, having the first peak during adolescence and the second peak in older adulthood ${ }^{[1]}$. The first peak is in the 10-14-year-old age group, coinciding with the pubertal growth spurt. This suggests a close relationship between the adolescent growth spurt and osteosarcoma. The second osteosarcoma peak is in adults older than 65 years of age; it is more likely to represent a second malignancy, frequently related to Paget's disease. The incidence of osteosarcoma has always been considered to be higher in males than in females, occurring at a rate of 5.4 per million persons per year in males vs. 4.0 per million in females, with a higher incidence in blacks (6.8 per million persons per year) and Hispanics (6.5 per million), than in whites (4.6 per million).

The tumor may be localized at the end of the long bone. Most often it affects the upper end of tibia or humerus, or lower end of femur. Osteosarcoma tends to affect regions around the knee in $60 \%$ of cases, $15 \%$ around the hip, $10 \%$ at the shoulder, and $8 \%$ in the jaw. The tumor is solid, hard, irregular ("fir-tree," "moth-eaten" or "sun-burst" appearance on X-ray examination) due to the tumor spicules of calcified bone radiating in right angles. These right angles form what is known as Codman's triangle. Surrounding tissues are infiltrated. The overall 5 -year survival rate for osteosarcoma is $68 \%$, without significant gender difference. The age of the patient is correlated with the survival, with the poorest survival among older patients. Complete surgical excision is important to ensure an optimum outcome. Tumor staging, presence of metastases, local recurrence, chemotherapy regimen, anatomic location, size of the tumor, and percentage of tumor cells destroyed after neo adjuvant chemotherapy have effects on the outcome.

\section{Case Report}

The patient is a 51-year-old male farmer who suffered acute onset of right knee pain one Friday in March after a long day of work. The pain persisted over the weekend becoming severe enough on Sunday night that it woke him from sleep. He was evaluated by his primary care physician the following morning and found on exam to have medial joint line tenderness. A possible intra-articular injury was suspected and the patient was referred our hospital for evaluation. Examination revealed pain with flexion of the knee beyond 90 degrees. Careful evaluation of the medial aspect of the knee revealed pain with palpation of the proximal tibia just adjacent to the medial joint line, but no actual joint line tenderness. Due to this discrepancy, radiographs were obtained, which showed a subtle radiolucent lesion in the proximal anteromedial tibial metaphysis (figs. 1 and 2). This was recognized as an abnormality and an MRI was ordered to more clearly define the lesion. 

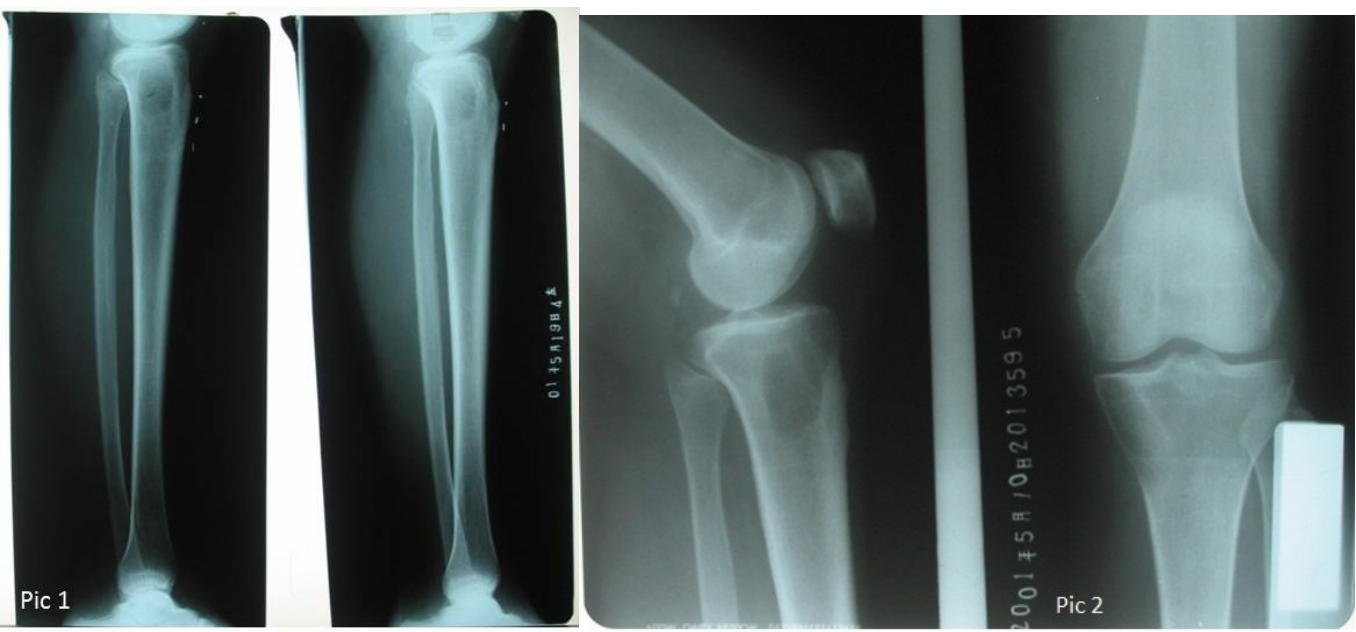

Figs 1 and 2: radiographs taken for the initial evaluation showed a subtle radiolucent lesion in the proximal anteromedial tibial metaphysis.

Radiographs showed the mass to involve the medial portion of the proximal tibial metaphysis. Staging studies, including computed tomography scanning of the chest and whole body bone scan, were done which did not show any evidence of metastatic disease to the lungs or other bony sites ${ }^{[3]}$. Hematological tests were within normal range except serum ALP was elevated (185; normal: 20 140 IU/L).

\section{Pathology and further workup}

Histologic evaluation of CT guided core biopsies of the lesion showed a spindled and epithelioid neoplasm with marked cytologic atypia and atypical mitotic forms. Close examination of the tumor showed, a pale-pink extracellular matrix that is diagnostic of unmineralized bone or osteoid ${ }^{[2]}$. The combination of the malignant cytology and the presence of osteoid are diagnostic of osteosarcoma.
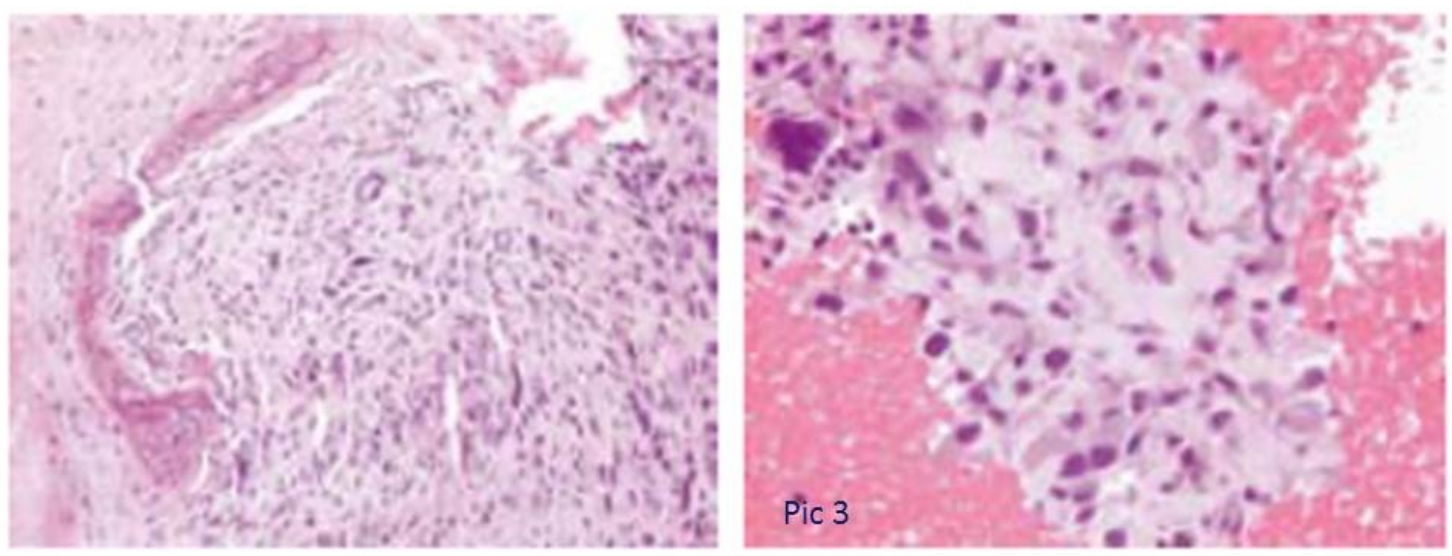

Fig 3: biopsies of the lesion (H\&E; 400X)

\section{Surgical treatment \\ Resection}

The tumor was approached through a medial incision extending from the mid-thigh along the medial femoral condylar line to the proximal 3rd of the tibia ${ }^{[4]}$. The biopsy site was kept in continuity with the resected specimen. The saphenous vein was identified, dissected and protected. The medial head of the gastrocnemius and soleus were retracted posteriorly. The soleus arch was divided while protecting the popliteal vessels. The pes anserine tendons and semimembranosus were divided, and the medial menisco-tibial ligament was divided anteriorly as far as the patellar tendon. The patellar tendon was preserved and retracted anteriorly [5]. The arthrotomy was extended posteriorly to the midline where the popliteal vessels were again identified and protected. Care was taken to preserve the entire medial meniscus. The anterolateral tibia was then exposed by raising a large anterolateral flap. Then, using C-arm control, K-wires were placed outlining the length and extent of our osteotomy. An oscillating saw and osteotomes were used to make the osteotomy. We had direct visualization of the articular surface at this point and placed the osteotome just medial to the tibial spines, 
preserving the insertion of the cruciates by undercutting of the tibial spines ${ }^{[6]}$. The osteotomies were completed, the specimen was passed off, and curettings were taken for a deep margin ${ }^{[9]}$.

\section{Reconstruction}

An osteoarticular autograft had been a center of attention for this reconstructive surgery. The whole patella was utilized to reconstruct the medial facet and meniscus of the tibia. The medial margin was reconstructed using a full thickness coronal iliac crest graft. After getting confirmation of exact margins, we sculpted the graft to fit the resection defect. The menisci from the grafts were trimmed, leaving a small rim peripherally. Multiple k-wires were used to hold the grafts in place. Sutures were then used to reattach the patient's native menisco- tibial ligament to the remaining rim of the graft menisci. The medial arthrotomy was then closed, and the pes anserine tendons, MCL and medial retinaculum were repaired [[7]] [[9]] [[10]]. The subcutaneous tissues were then closed in layers, the wounds were dressed, and the patient was placed in a long leg anterior and posterior plaster of Paris (POP) slabs.

\section{Post op}

Post-operative fluids and chemotherapy were started immediately after. Post op labs were checked and vitals were closely monitored in indoor for 2 weeks. Sutures were removed after that. The skin at the site of the surgical scar was healthy. Non-weight-bearing ambulation began after 2 weeks.

Repeat computed tomography (CT) scan of the chest was normal. The POP slabs were removed after 6 weeks and a custom made above knee caliper with a hinge joint was worn for 3 months. Three months after surgery, the patient was advised to start partial weight-bearing with a walker ${ }^{[11]}$. The patient is now walking full weight-bearing and doing all of his normal activities himself.

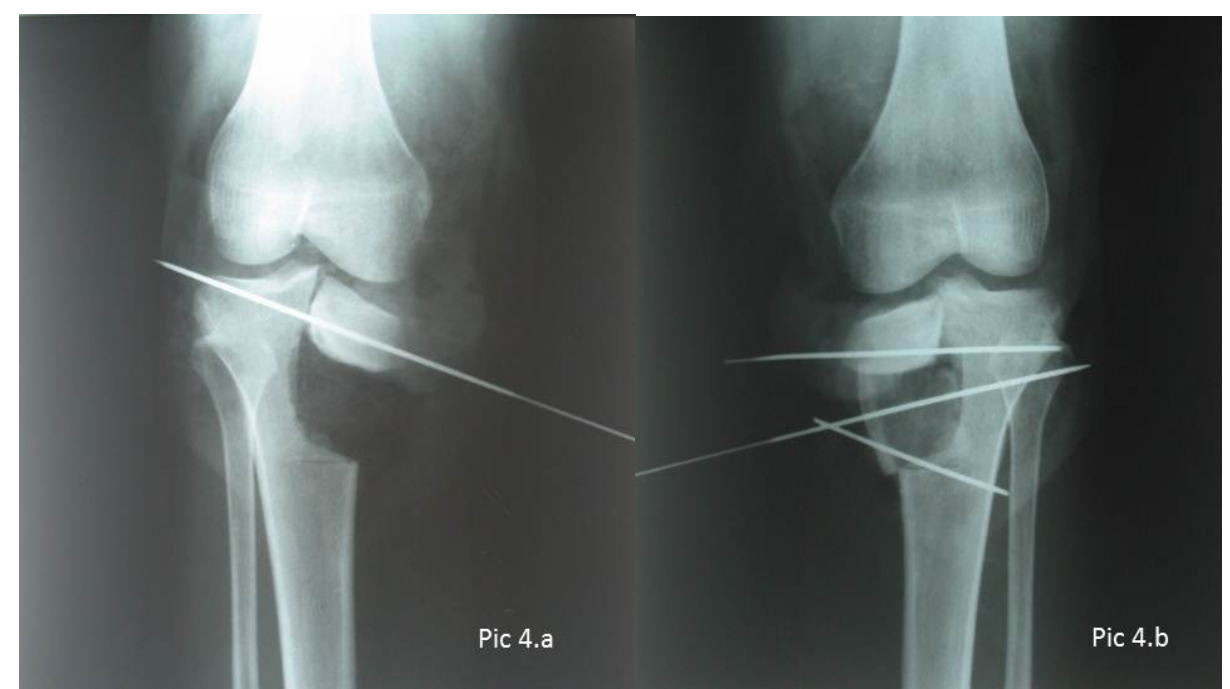

Figs 4(a and b): reconstructions of resection defects with patella (pic 4.a) and then full thickness iliac grafts (pic 4.b)

Follow ups:

1) 6 month follow up:

The patient was then allowed to walk weight-bearing according to his tolerance.

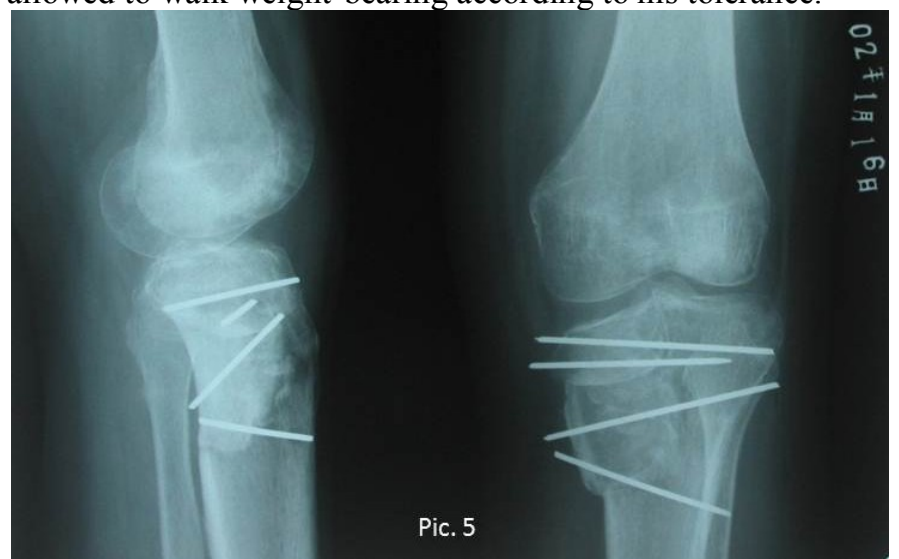

Fig 5: radiographs showing hard callus formation and mineralization 
2) One year follow up:

An excellent ossification and ongoing remodeling was revealed by radiographs. Second-step surgery was performed and all K-wires were taken out under spinal anesthesia (SAB).

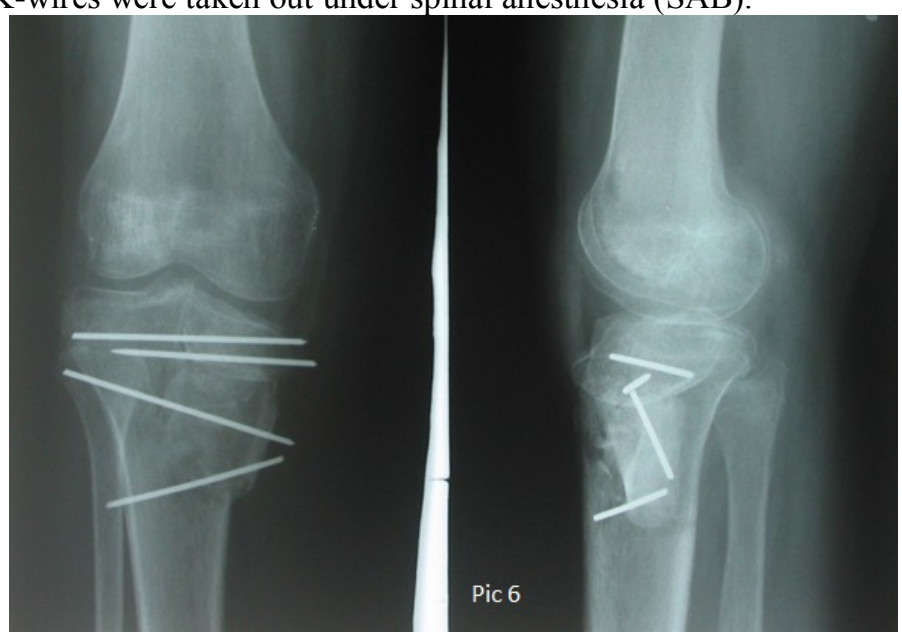

Fig 6: radiograph after 1 year before second surgery

3) One and half year follow-up:

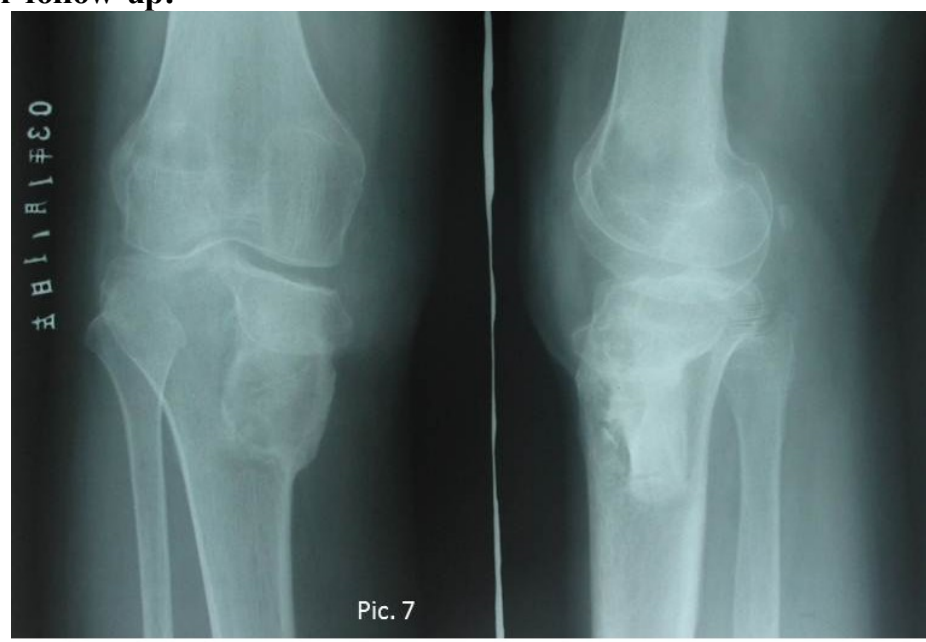

Fig 7: mineralization and remodeling after removal of K-wires

4) 3 and half year follow up:

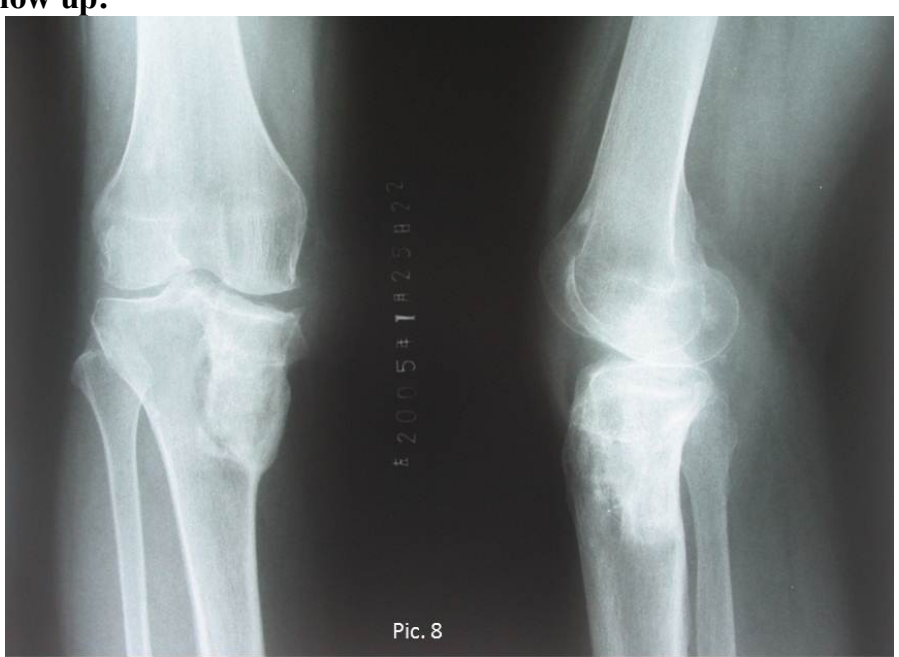

Fig 8: radiographs show fine remodeling in articular and plateau areas 


\section{Outcome:}

The condition of the limb at the latest follow-up examination was evaluated thoroughly. Physical examination revealed that the arc of active motion of the knee ranged 35 to 175 degrees and the findings relative to muscle strength about the knee were encouraging.

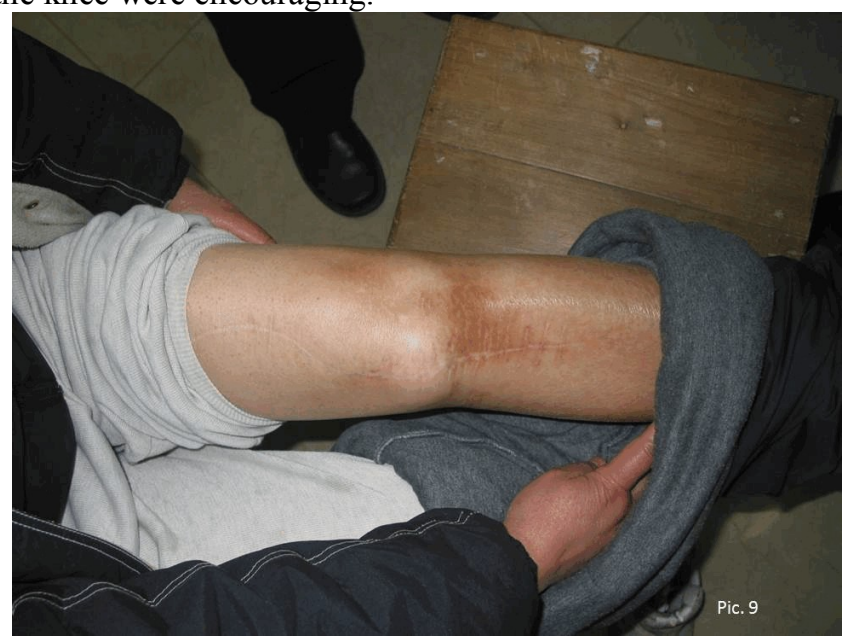

Fig 9: patient showing his knee during follow-up examination

Also the strength of knee extension was better than antigravity. He had no difficulties performing his normal daily activities. Chest radiographs taken during each follow ups were non-suggestive for any carcinomatous changes. Serum ALP level decreased considerably after $1^{\text {st }}$ surgery and never exceeded the upper range in subsequent tests.

\section{Discussion}

Current management of osteosarcoma requires a multidisciplinary approach including a team of orthopedic oncologists, radiologists and pathologists. The goal of treatment is to achieve local resection of the tumor and systemic control of the disease with acceptable functional recovery ${ }^{[7]}$. Recently, few reports have demonstrated that proximal tibial osteoarticular autograft reconstruction compare favorably to allografts and metal endoprosthetic reconstruction in this location, and probably have improved long term function because of the ability to salvage the patellar tendon attachment. In the described resection, the majority of the functional components of the joint are preserved, including not only the anterior and posterior cruciate ligaments, but also the lateral collateral ligament and greater than $50 \%$ of the articular cartilage of the tibial plateau [[11]]. Presumably this joint preservation will translate into further improvements in graft survival and long-term function. Although it remains to be seen whether there is additional risk in terms of local recurrence with this type of resection, these potential benefits must be considered. Worth mention here, the era this surgery was performed, no CT/MRI was available at our hospital for further evaluation.

Autograft tissue is the safest and fastest-healing tissue that can be used. In our case, we used the whole patella that was the nearest donor site with adequate blood supply, a must for predicted outcome. In terms of autograft incorporation, multiple current studies suggest that autograft incorporation in the metaphyseal region is faster when compared to the diaphyseal region. Autograft reconstruction in metaphyseal osteotomies heal at an average of 3 months compared to 6 months for allografts and a range of 9 to 18 months for diaphyseal osteotomies $^{[[4]][13]}$

The following table justifies our selection for Autograft for this particular case i.e. we achieve all osteoconductive, osteoinductive and osteogenic benefits from Autograft.

\begin{tabular}{|l|l|l|l|}
\hline Table 1: Properties of various types of bone graft sources. \\
\hline & Osteoconductive & Osteoinductive & Osteogenic \\
\hline Alloplast & + & - & - \\
\hline Xenograft & + & - & - \\
\hline Allograft & + & $+/-$ & - \\
\hline Autograft & + & + & + \\
\hline
\end{tabular}

\section{Conclusion}

In this age of total knee replacement or a prosthetic leg, we favor our promising autograft reconstruction over any other methods. Current reports have demonstrated that proximal tibial osteoarticular autograft reconstruction compare favorably to allografts and metal endoprosthetic reconstruction in this location, 
and probably have improved long term function for all age patients and also in non-favored knee replacement surgeries. Hope a number of clinical trials would run and more specifications will be published in coming days.

\section{References}

[1]. Ottaviani G, Jaffe N. (2009): The epidemiology of osteosarcoma. Cancer Treat Res. 152:3-13.

[2]. Atesok KI, Alman BA, Schemitsch EH, Peyser A, Mankin H: Osteoid osteoma and osteoblastoma: J Am Acad Orthop Surg. 2011 Nov; 19(11):678-89.

[3]. Harris IE, Leff AR, Gitelis S, Simon MA: Function after amputation, arthrodesis, or arthroplasty for tumors about the knee (1990): J Bone Joint Surg Am. 1990 Dec; 72(10):1477-85.

[4]. Campanacci M, Costa P (1979): Total resection of distal femur or proximal tibia for bone tumours. Autogenous bone grafts and arthrodesis in twenty-six cases: J Bone Joint Surg Br. 1979 Nov; 61-B (4):455-63.

[5]. Barrett GR, Noojin FK, Hartzog CW, Nash CR. (2002): Reconstruction of the anterior cruciate ligament in females: A comparison of hamstring versus patellar tendon autograft: Arthroscopy. 2002 Jan; 18(1):46-54.

[6]. Renard AJ, Veth RP, Schreuder HW, et a.(2000)1: Function and complications after ablative and limb-salvage therapy in lower extremity sarcoma of bone. J Surg Oncol 73(Suppl):198-205, 2000.

[7]. Lewis VO, Gebhardt MC, Springfield DS: Parosteal osteosarcoma of the posterior aspect of the distal part of the femur (2000). Oncological and functional results following a new resection technique: J Bone Joint Surg Am. 2000 Aug;82 -A(8):1083-8.

[8]. Sim, F. H.; Beauchamp, C. P.; and Chao, E. Y. S. (1987): Reconstruction of musculoskeletal defects about the knee for tumor. Clin. Orthop., 221:188-201, 1987

[9]. Aune, A. K., Holm, I., Risberg, M. A., Jensen, H. K. and Steen, H. (2001) Four-strand hamstring tendon autograft compared with patella-tendon-bone autograft for anterior cruciate ligament reconstruction: A randomized study with 2-year follow-up. American Journal of Sports Medicine 29, 722-728.

[10]. Enneking WF, Kagan A (1975): "Skip" metastases in osteosarcoma. Cancer 1975, 36:2192-2205.

[11]. James H. Beaty, MD S. Terry Canale: Canale \& Beaty (2007): Campbell's Operative Orthopaedics, 11th ed. Arthroplasty of the knee (Ch. 6), preoperative evaluation: Mosby, An Imprint of Elsevier: International Edition ISBN: 978-0-8089-2361-9.

[12]. Rue JP, Busam ML, Detterline AJ, Bach BR Jr.: Posterior wall blowout in anterior cruciate ligament reconstruction: avoidance, recognition, and salvage: J Knee Surg. 2008 Jul; 21(3):235-40.

[13]. Puri A1, Gulia A, Jambhekar N, Laskar S. The outcome of the treatment of diaphyseal primary bone sarcoma by resection, irradiation and re-implantation of the host bone: extracorporeal irradiation as an option for reconstruction in diaphyseal bone sarcomas. J Bone Joint Surg Br. 2012 Jul; 94(7):982-8. doi: 10.1302/0301-620X.94B7.28916. 